\title{
Site-Specific DNA Functionalization through the Tetrazene-Forming Reaction in lonic Liquids
}

Seiya Ishizawa, Munkhtuya Tumurkhuu, Elizabeth J. Gross, Jun Ohata*

Department of Chemistry, North Carolina State University, Raleigh, NC 27695

\begin{abstract}
Development of multiple chemical tools for deoxynucleic acid (DNA) labeling has facilitated wide use of their functionalized conjugates, but significant practical and methodological challenges remain to achievement of site-specific chemical modification of the biomacromolecule. As covalent labeling processes are more challenging in aqueous solution, use of nonaqueous, biomolecule-compatible solvents such as an ionic liquid consisting of a salt with organic molecule architecture, could be remarkably helpful in this connection. Herein, we demonstrate site-specific chemical modification of DNAs through a tetrazene-forming amine-azide coupling reaction using an ionic liquid. This ionic liquid-enhanced reaction process has good functional group tolerance and precise chemoselectivity, and enables incorporation into DNA of various useful functionalities such as biotin, cholesterol and fluorophores which could be incorporated into DNA through this method. A site-specifically labeled single stranded nucleotide, or aptamer interacting with a growth factor receptor (Her2) was successfully used in the fluorescence imaging of breast cancer cell lines. The non-traditional mediumpromoted labeling strategy described here provides an alternative design paradigm for future development of chemical tools for applications involving DNA functionalization.
\end{abstract}

\section{Introduction}

Chemically functionalized deoxyribonucleic acid (DNA) have become invaluable in various applications such as molecular beacons, ${ }^{1,2}$ polymerase chain reaction (PCR) technologies, ${ }^{3}$ deoxyribozymes (DNAzymes), ${ }^{4,5}$ asymmetric catalysis, ${ }^{6}$ and therapeutics ${ }^{7}$ such as antisense sequences, ${ }^{8,9}$ transcription-factor decoys, ${ }^{10,11}$ and CpG motifs. ${ }^{12,13}$ The methods of chemical synthesis of DNA have significantly facilitated the introduction of desired functionalities onto a specific position, ${ }^{14}$ and post-synthetic modification methods are also powerful chemical tools, especially for labeling of large DNA targets. ${ }^{15-18}$ Bioinspired enzymatic post-synthetic labeling technologies have emerged as useful approaches through sequence- or structure-dependent substrate recognition, ${ }^{19}$ and chemical modification is an alternative means for site-specific DNA labeling by incorporation of a chemical handle that is inert toward endogenous chemical functional groups but reactive toward a labeling reagent. ${ }^{20,21}$ However, despite the remarkable advance of the bioconjugation technologies during the past decades, site-specific chemical modification remains a serious challenge due to its demanding criteria which include adequate reactivity of the labeling reagents toward the target site and high selectivity in biomolecule-compatible solvents such as aqueous buffer. Finding the appropriate balance between the reactivity and selectivity of a labeling reagent requires careful, time-consuming screening, and the need for an aqueous solution renders the realization of selective DNA bioconjugation even more problematic, as development of organic reactions in aqueous media is difficult. 22

Bioconjugation processes based on ionic liquids represent an alternative strategy to address the compatibility issue between DNAs and organic chemical reactions. Owing to the ionic nature and solubility of DNAs, water or aqueous buffer is often a common choice for bioconjugation processes, although aqueous reaction conditions are not preferable from the perspective of the reaction because of their negative effects, which include the solubility and stability of labeling reagents and sluggish kinetics. As an alternative, ionic liquids, compounds completely composed of ions have organic frameworks with a constant charge state and have been successfully employed for dissolution and stabilization of various DNA molecules. ${ }^{23,24}$ However, ionic liquids have not been employed in DNA bioconjugation processes even though their modular properties suggest they may be preferable for both the DNA and labeling processes. 
In this Article, we report that a tetrazene forming reaction based on an ionic liquid enables sitespecific labeling of the alkylamine tag on a desired position of DNA (Fig. 1). Our research program (Bioconjugation In Nonaqueous-Driven Reaction Solvent or BINDRS) to develop novel bioconjugation using nontraditional media, recently discovered that triphenylphosphine promotes a coupling reaction between an azide and an alkylamine in peptides or proteins, forming a tetrazene group with four consecutive nitrogen atoms. ${ }^{25}$ With no noticeable effect on the target peptides or proteins, this reaction, which takes place in an ionic liquid, displays excellent chemoselectivity for alkylamines in the presence of a variety of other $\mathrm{NH}$ groups in for example, guanidine or imidazole. The tetrazene formed in this reaction serves as a linker which can survive $\mathrm{pH}$ changes and redox mediators. We have found that the phosphine-azide reaction has virtually no effect on native DNA functional groups such as DNA bases, phosphate backbone and ribose groups. This capability presents the possibility of site-specific modification at a desired location in DNA through incorporation of an exogenous alkylamine group. Reagent screening revealed the wide tolerance of the phosphine-azide reaction of various chemical functionalities, enabling preparation of a variety of functionalized DNA aptamers such as fluorophores and cholesterol and biotin conjugates. The aptamer conjugates were successfully used in staining experiments of cancer cell lines, validating the compatibility of the ionic liquid-based modification processes with biomolecules such as DNA.

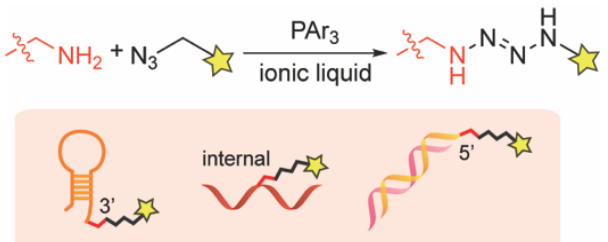

Fig. 1. Site-specific tetrazene-forming reaction on DNA substrates.

\section{Results and discussion}

Our initial survey of the reactivities of the adenine (A), thymine (T), cytosine $(C)$, guanine $(G)$, and uracil (U) nucleotides in a simple DNA substrate revealed their inertness toward the phosphine-azide coupling reaction (Fig. 2). In order to understand the applicability of the phosphine-azide coupling reaction, we first applied nonfunctional azide reagent (1a) to a tetrathymidine containing an additional nucleotide at the $5^{\prime}$ terminus (Figs. 2A-2C). Notwithstanding the presence of different types of $\mathrm{NH}_{2}$ groups in native DNAs, only traces of tetrazene-modified DNAs were observed in mass spectrometric analysis after the reaction and subsequent buffer exchange processes (Figs. 2D-2H). On the other hand, introduction of alkylamine at the 5 '-terminus of the backbone led to a significant increase in the signal from the peak corresponding to the formation of the tetrazene group (Fig. 2I). Those results suggest that the ionic liquid-based phosphine-azide reaction has a high specificity for alkylamines over other endogenous arylamine groups. 


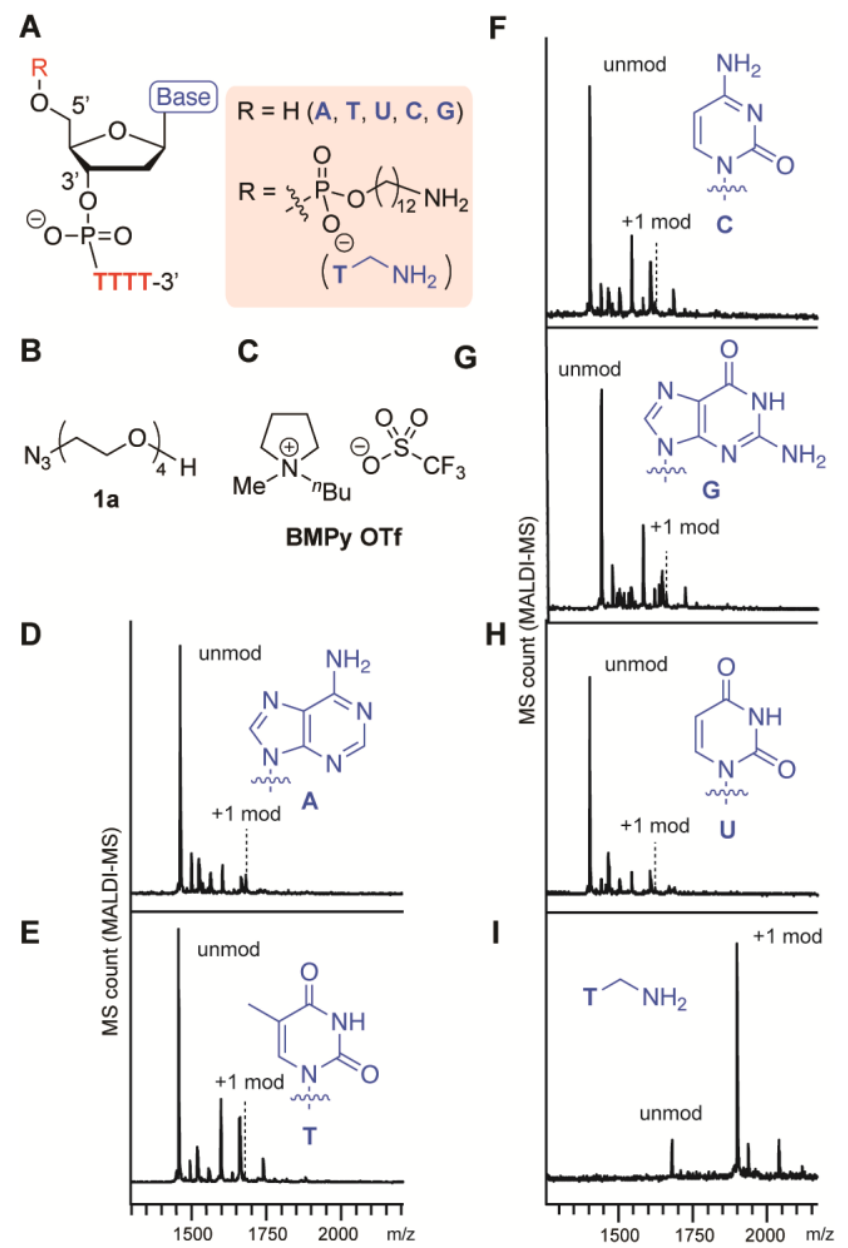

Fig. 2. Alkylamine-selective tetrazene-forming reaction with pentanucleotides in an ionic liquid. Reaction conditions: Reaction conditions: $\mathrm{KHCO}_{3}(20 \mathrm{mM})$, XTTTT $(0.2 \mathrm{mM})$, azide 1a $(7.5 \mathrm{mM})$, and $\mathrm{PPh}_{3}(20 \mathrm{mM})$ in 1-butyl-1methylpyrrolidinium trifluoromethanesulfonate (BMPy OTf) at $50{ }^{\circ} \mathrm{C}$ for $2 \mathrm{~h}$. (A) Chemical structure of nucleotide backbone in an XTTTT sequence with or without an alkyl amine. (B) Chemical structure of the azide (1a). (C) Structure of the ionic liquid, BMPy OTf. (D-I) Matrix-assisted laser desorption/ionization (MALDI-MS) analysis of the reaction of XTTTT where $X=$ adenosine $(D)$, thymidine $(E)$, cytosine $(F)$, guanosine $(G)$, deoxyuridine $(H)$, and thymidine $(T)$ with alkylamine (I) containing a 12 carbon linker (5AmMC12).

The high functional group tolerance of the modification process allowed incorporation of a variety of alkyl azide reagents to the alkyl amine-tagged DNA (Figs. 3 and S1). The trimethyl-silyl (TMS) group has been used increasingly as a chemical reporter in structural biology research because of its characteristic chemical shift in nuclear magnetic resonance (NMR) spectra. ${ }^{26,27}$ Despite the relatively large steric bulk of the trimethylsilyl group, the TMS-methylazide (1) could be used to introduce the TMS group to the DNA. Tertiary amine-containing reagents such as $\mathbf{1 c}$ or $\mathbf{1 d}$ did not decrease the efficiency of the modification, and could be useful for introduction of an additional positive charge to the biomolecule. Incorporation of the morpholine group (1d) is noteworthy as the resulting tag could be useful in endoplasmic reticulum-targeting applications. ${ }^{28}$ Aromatic rings and a fluorophore scaffold are also compatible with the modification, enabling preparation of a color-palette of DNA-fluorophore conjugates. Interestingly, despite the versatile reactivities of azides and phosphine, and their derivatives, such as phosphazide and iminophosphorane, a single product was observed with an alkyl bromide substituted azide (19) without loss of the bromide group. As the incorporation of the dimethylalkyl bromide is common in radical polymerization processes, ${ }^{29-31}$ the successful attachment of the group assists further development of DNA-polymer conjugates. Thus, the 
phosphine azide reaction in not affected by a series of functional groups such as silyl, tertiary amino, ether, ester, aryl alcohol, tertiary alkyl bromide and alkene groups.

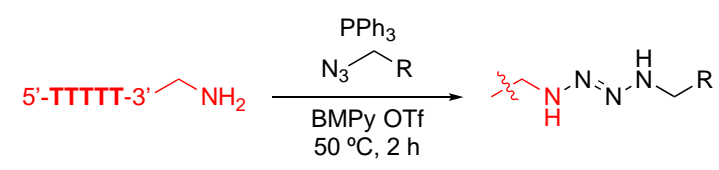<smiles>C[Si](C)(C)CN</smiles>

$$
\text { 1b }(73 \%) \quad \text { 1c }(94 \%) \quad \text { 1d }(98 \%) \quad \text { 1e }(97 \%)
$$<smiles>CC(C)(Br)C(=O)OCCN</smiles>

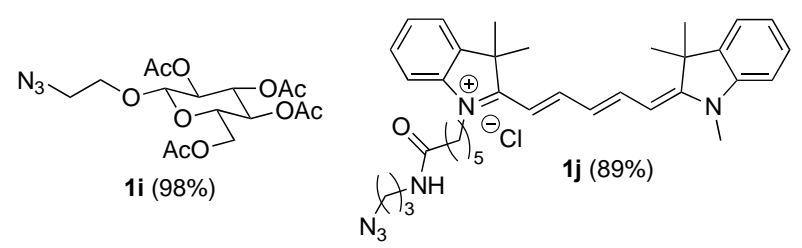

Fig. 3. Tetrazene-forming DNA bioconjugation with a variety of alkyl azides. Modification reaction conditions: $\mathrm{KHCO}_{3}$ (20 mM), 5'-TTTTT-3'-alkyl-NH 2 (0.2 mM), azide derivatives 1b-1j (7.5 mM), and PPh $(20 \mathrm{mM})$ in BMPy OTf at $50{ }^{\circ} \mathrm{C}$ for $2 \mathrm{~h}$. * Reaction was incubated overnight. Conversion rates in the parentheses were calculated based on matrixassisted laser desorption/ionization (MALDI-MS) analysis.

In an effort to understand the compatibility of the functional groups and enhance the reaction efficiency, we tested different types of phosphine and phosphite reagents (Fig. 4). A DNA aptamer for human serum albumin was chosen as a model substrate for this study of phoshines. ${ }^{32}$ We focused only on reasonably air-stable phosphines including triaryl-phosphines with a range of substituents (2a-2g), alkylphosphine (JohnPhos, $\mathbf{2 h}$ ), and aryl phosphite (2i) and used triphenylphosphine oxide (3) as a negative control (Figs. 4A,4C). ${ }^{33}$ The degree of the modification reaction was assessed with biotin-azide which provides an analysis handle for anti-biotin southern blotting with a streptavidin-fluorophore conjugate. With the sole exception of tris(pentafluorophenyl)phosphines (2b), all the triarylphosphines $(\mathbf{2 a}, \mathbf{2 c}-\mathbf{2 g})$ displayed a fluorescence signal at a similar level, indicating the minor effect of the subtle changes of the electronic properties of the aryl substituents (Figs. 4D, S2). On the other hand, substantially weaker fluorescence signals were observed from reactions involving bisalkyl-monoarylphosphine (2h) or triphenylphosphite (2i), which suggests the necessity of triarylphosphine for the reaction. In order to visualize the total DNA amount on a blot membrane, Mayer's hemalum solution (Fig. S3), which relies on coordination of aluminum with the phosphate backbone was employed. ${ }^{34}$ In contrast to the southern blot showing a varied fluorescence signal dependent on the biotin attachment on DNA, Mayer's hemalum stain showed similar intensity across all the conditions, confirming the validity of the experimental design (Fig. S2). The triarylphosphinedependent modification was also confirmed by mass spectrometry (Fig. S2). We also found that different types of ionic liquid could be utilized for this chemical transformation (Figs. 4B, S4). Furthermore, double-stranded DNAs with the alkylamine tag exhibited comparable reactivity to the single-stranded DNA system as well (Fig. S5). 
A

C

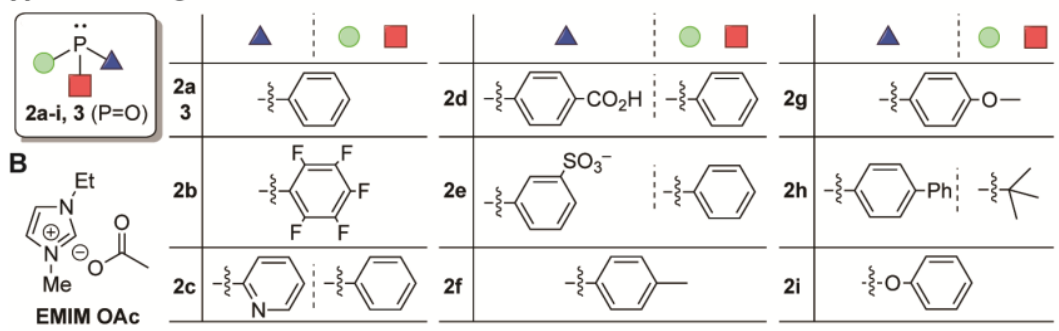

D

Fig. 4. Screening of phosphine reagents on tetrazene formation reaction on a DNA aptamer toward human serum albumin (HSA). Sequence of the HSA aptamer: 5'-TGCGGTTGTAGTACTCGTGGCCG-3'. ${ }^{32}$ Reaction conditions: HSA DNA aptamer $(0.1 \mathrm{mM}), \mathrm{KHCO}_{3}(20 \mathrm{mM})$, biotin azide $(7.5 \mathrm{mM})$, and phosphine $(3.0 \mathrm{mM})$ in a mixture of 1-ethyl-3methylimidazolium acetate (EMIM OAC)/DMF (1:1) at $50{ }^{\circ} \mathrm{C}$ for $2 \mathrm{~h}$. (A) General chemical structure of phosphine. Green circle, red square and blue triangle each represents the aryl or alkyl groups shown in Fig. 5C. (B) Chemical structure of ionic liquid 1-ethyl-3-methylimidazolium acetate, EMIM OAc. (C) Chemical structures of the aryl or alkyl groups of different phosphine/phosphite reagents. (D) Bar graph showing the anti-biotin southern blot after modification of the HSA DNA aptamer with biotin azide with different phosphines. Error bars represent standard deviation $(n=3)$. Representative blot membrane images for the anti-biotin southern blot (Cy5) and total stain with Mayer's hemalum solution (MHS) are shown below the bar graphs. Full-width southern blot membrane images are shown in Fig. S2.

The site-specific incorporation of the tetrazene functional group proved to be independent of the alkylamine introduction site (Figs. 5A, 5B). In order to determine if the reactivity of the alkylamine group is affected by its location on the DNA sequence, we tested the reaction of a small DNA (TTTTT) with an alkylamino group on the internal thymidine (alkylamine on thymidine base), 3 ' terminus, and 5 '-terminus (Fig. 5A). Assessment of the modification was performed by using fluorophore-azide (1k, boron dipyrromethene, BODIPY) to compare the reaction efficiency of the different DNAs (Fig. 5B), because the fluorescence intensity from the DNAs after the reaction reflects the efficacy of the reaction process. The DNAs were subjected to the reaction cocktails containing the fluorophore azide (1k) and triphenylphosphine, and the reactions were quenched at a different time points $(0,30$ and $60 \mathrm{~min}$ ). After the buffer exchange process to remove the ionic liquid and excess reagents, the fluorescence intensities of the DNA were visualized on a nylon blot membrane (Figs. 5A and S6). There is a slight difference of the labeling efficiency tendency, but we did not observe substantial difference of the reactivity at any position, and the negative control without amine groups displayed no meaningful reactivity. The similar observation was also confirmed by MALDI-MS analysis (Fig. S6), and this demonstrated the generality of the reactions on different location of alkylamines.

Taking advantage of the minimal reactivity of the phosphine-azide toward endogenous DNA functional groups, we were able to selectively modify alkylamine-labeled DNA in a mixture with other untagged DNAs (Fig. 5C). Thymidine pentamer (TTTTT) with and without the alkylamine tag at the 5' terminus was incubated with BODIPY-azide (1k) and triphenyl-phosphine in the presence of a series of DNAs ranging from 10 to 300 base pairs. Agarose gel analysis showed a single fluorescence band at the bottom of the gel representing the alkylamine-containing condition. In contrast, a total stain of the gel with a SYBR fluorophore confirmed the presence of a number of DNAs, demonstrating that the modification process indeed occurred preferentially on the alkylamine-tagged DNA over other untagged DNAs. 


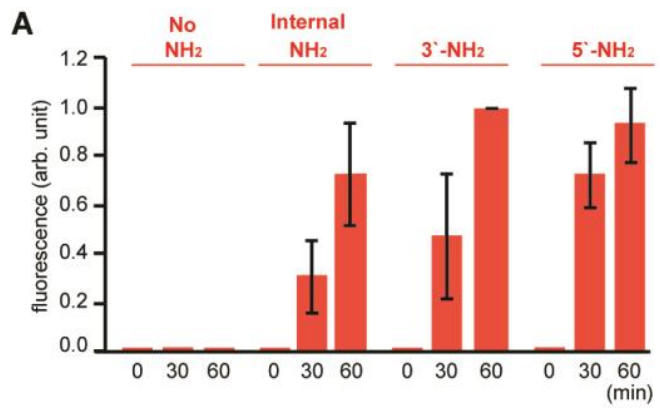

B
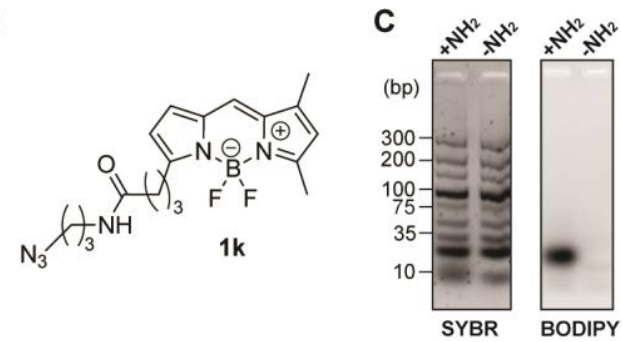

Fig. 5. Reactivity and selectivity analysis of the tetrazene forming reaction on pentanucleotides with an alkylamine in different locations. (A) Bar graph showing fluorescence intensity of 5'-TTTTT-3' with and without an alkyl amine and treated with fluorophore azide 1k. Reaction conditions: $\mathrm{KHCO}_{3}(20 \mathrm{mM}), 5^{\prime}-\mathrm{TTTTT}-3^{\prime}$ with and without amine group $(0.2 \mathrm{mM})$, azide $\mathbf{1 k}(3 \mathrm{mM})$, and $\mathrm{PPh}_{3}(3 \mathrm{mM})$ in BMPy OTf at $50{ }^{\circ} \mathrm{C}$ for a specific time. Error bars represent standard deviation ( $n=3$ ). (B) Chemical structure of alkylazide containing a boron dipyrromethene (BODIPY) group (1k). (C) Agarose gel images for the reaction of TTTTT-5'- $\mathrm{NH}_{2}$ with azide $\mathbf{1} \mathbf{k}$ in the presence of DNA ladder (10-300 bps). Total DNA samples were visualized by the fluorescence from SYBR Gold nucleic acid gel stain (Cy3 excitation and emission), while modified DNA samples were visualized by the fluorescence from BODIPY (Cy2 excitation and emission). Reaction conditions: $\mathrm{KHCO}_{3}(20 \mathrm{mM})$, TTTTT with or without $\mathrm{NH}_{2}$ tag at $5^{\prime}$ position $(0.2 \mathrm{mM})$, DNA mixture $(0.9 \mathrm{mg} / \mathrm{mL})$, azide 1k (3 mM), and $\mathrm{PPh}_{3}(3 \mathrm{mM})$ in BMPy OTf at $50^{\circ} \mathrm{C}$ for $2 \mathrm{~h}$.

The ionic liquid-based approach also enabled the introduction of a hydrophobic anchor onto a DNA aptamer (Fig. 6). Synthetic DNAs with hydrophobic tags have been studied increasingly as hydrophobic DNAs exhibit unique properties such as formation of nanostructures and cell permeability for molecular transport across cell membranes. ${ }^{35,36}$ However, due to the strong preference of an aqueous or highly polar solution of DNA with its polyionic nature, introduction of nonpolar, hydrophobic groups including lipids and steroids is chemically challenging. With the ionic nature of the hydrophobic scaffold of ionic liquids, we hypothesized that our BINDRS strategy could address the dilemma of a reaction solvent incorporating a hydrophobic tag reacting with hydrophilic DNA molecules. A DNA aptamer toward the RNA hairpin of human immunovirus (HIV)-1 transactivation-responsive (TAR) element ${ }^{37}$ was incubated with cholesterol azide (1I) and different types of phosphine reagents (Fig. 6a). Consistent with the known cholesterol-dependent aggregation of DNA ${ }^{38}$ as well as with the phosphine screening experiments in Fig. 5, the agarose gel analysis showed the aggregation of the DNAs with active triarylphosphine reagents in a high conversion but not from sterically bulky alkylphosphines nor from phosphine oxide negative control conditions (Fig. $6 \mathrm{~B})$. Even though the polarity or solubility of DNAs would have been altered by the cholesterol modification processes, no significant loss of nucleotides was observed after the buffer exchange, as confirmed by results from the use of Mayer's hemalum stain (Fig. S7). The high efficiency of the modification process with cholesterol was confirmed by MALDI-MS too (Fig. 6C). The aggregation observed in the agarose gel is indicative of the potential capability of the aptamer modified with a hydrophobic tag to diffuse into the cell membrane, and accordingly, we tested the cholesteroltagged aptamer in confocal microscope experiments using a cancer cell line. Cultured HeLa cells were incubated with the cholesterol aptamer hybridized with its complementary sequence bearing a cyanine fluorophore (Cy5), and the fluorescence signal was visualized on a confocal microscope (Fig. 
6D). As anticipated, ${ }^{39}$ the fluorescence signal derived from the Cy5 fluorophore was observed to be dependent on the modification. Staining of the actin filament with a phalloidin-fluorophore conjugate shows the presence of cells in both conditions. Together, these experiments demonstrate the successful introduction of cholesterol to the aptamer sequence through the ionic liquid-based reaction.

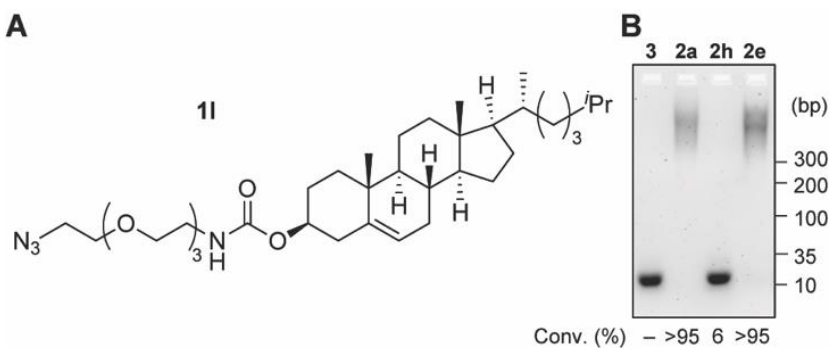

C

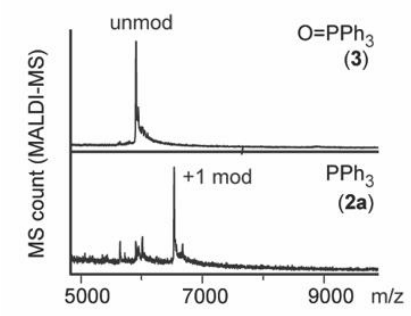

D

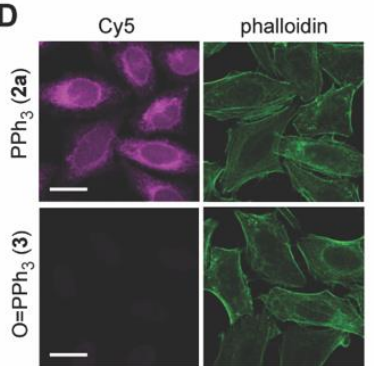

Fig. 6 Attachment of cholesterol to a DNA aptamer toward the RNA hairpin of human immunovirus (HIV)-1 transactivation-responsive (TAR) element through the tetrazene-forming reaction. Sequence of the HIV-1-TAR aptamer: 5'-CCCTAGTTAGCCATCTCCC-3'. ${ }^{37}$ Modification conditions: HIV-1-TAR-5'-NH aptamer (0.1 mM), $\mathrm{KHCO}_{3}(20$ $\mathrm{mM})$, azide $1 \mathrm{l}(7.5 \mathrm{mM})$, and $\mathrm{PPh}_{3}(2)$ or $\mathrm{O}=\mathrm{PPh}_{3}(3)(20 \mathrm{mM})$ in EMIM OAc/DMF/DMSO (2:1:1) at $50{ }^{\circ} \mathrm{C}$ for $2 \mathrm{~h}$. (A) Chemical structure of cholesterol azide (11). (B) Agarose gel analysis of HIV-1-TAR-5'-NH 2 aptamer modified with cholesterol azide (1) in the presence of triphenylphosphine oxide (3), triphenylphosphine (2a) JohnPhos (2h), and sulfonate-substituted triphenylphosphine (2e). Degree of conversion was calculated by quantification of the unmodified DNA bands in comparison with the triphenylphosphine oxide condition as $100 \%$ of the starting material. (C) Matrix-assisted laser desorption/ionization (MALDI-MS) analysis of the modification of HIV-1-TAR-5' NH$_{2}$ aptamer with azide $\mathbf{1 l}$ and triphenylphosphine oxide (top, negative control) or triphenylphosphine (bottom). (D) Confocal microscopy images of HeLa cells stained with HIV-1-TAR aptamer hybridized with its complementary DNA-Cy5 conjugate (magenta). Top: Cholesterol-modified aptamer (azide/PPh 3 -treated aptamer). Bottom: Unmodified aptamer (azide/O=PPh -treated aptamer). Green: Actin filament stain with Phalloidin-CF488 conjugate. Scale bar: 20 $\mu \mathrm{m}$.

Finally, the ionic liquid-based phosphine-azide reaction was applied to labeling of a therapeutically important DNA aptamer (Fig. 7). The Her2 receptor is an epidermal growth factor related protein (ErbB2) family of receptor tyrosine kinases and an emblematic example of overexpressed proteins in several types of cancer cells (Fig. 7A). ${ }^{40}$ We have adopted sequence (42 nucleotides) of a DNA aptamer to the Her2 receptor originally reported by Yarden et al ${ }^{41}$ Modification of the Her2 aptamer with biotin-azide reagent proceeded smoothly in the same reaction conditions as those used with other aptamers, and was confirmed by MALDI-MS and anti-biotin southern blotting (Fig. S8). A gel shift assay using streptavidin also demonstrated the consumption of the unmodified aptamer (Fig. S9). We used the biotinylated aptamer for cell imaging experiments on an Her2-overexpressing cell line, SK-BR-3. Treatment of SK-BR-3 cells with the biotinylated aptamer gave the streptavidinfluorophore (Cy5) conjugate which displayed strong fluorescence signals while negligible fluorescence was observed from the unconjugated aptamer (Fig. 7B). Importantly, cells treated with the biotinylated aptamer hybridized with its complementary sequence showed substantial decrease in the fluorescence signals, showing the importance of the single-strand aptamer structure for its 
binding with the target protein. As such, we have confirmed the conservation of the aptamer's binding capability toward antigen targets even after treatment with ionic liquids and phosphine/azide reagents, demonstrating the practical utility of the ionic liquid-DNA bioconjugation approach.

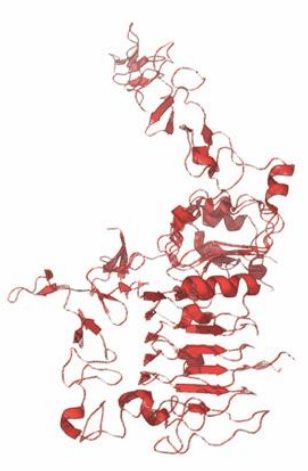

B

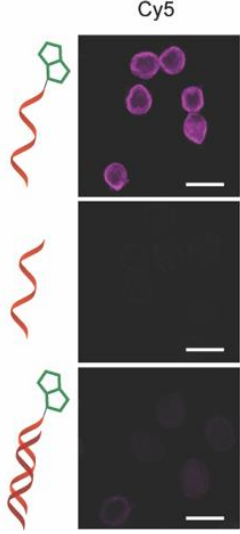

DAPI

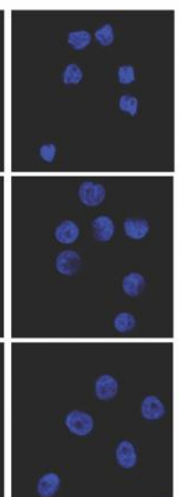

DIC

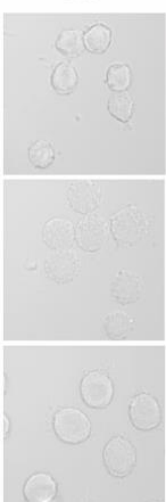

Fig. 7. Tetrazene-forming reaction of DNA aptamer with the Her2 receptor. Sequence of the Her2 aptamer: $5^{\prime}-$ GCAGCGGTGTGGGGGCAGCGGTGTGGGGGCAGCGGTGTGGGG-3' ${ }^{41}$ Modification conditions: Her2 aptamer-5'-NH ${ }_{2}$ aptamer $(0.1 \mathrm{mM}), \mathrm{KHCO}_{3}(20 \mathrm{mM})$, biotin azide $(7.5 \mathrm{mM})$, and $\mathrm{PPh}_{3}(20 \mathrm{mM})$ in EMIM OAc/DMF/DMSO (2:1:1 ratio) at $50{ }^{\circ} \mathrm{C}$ for $2 \mathrm{~h}$. (A) Partial crystal structure of Her2 receptor (PDB ID: 1N8Z). (B) Confocal microscopy images of Her2overexpressing SK-BR-3 cells stained with biotin-modified Her2 aptamer (top), unmodified aptamer (middle), or biotin-modified Her2 aptamer hybridized with complementary DNA sequence (bottom). The bound aptamer was visualized by streptavidin-fluorophore (Cy5) conjugate (1:50 dilution) shown in magenta. Blue: Nuclear stain with DAPI. DIC: differential interference contrast. Scale bar: $20 \mu \mathrm{m}$.

\section{Conclusion}

The ionic liquid-based tetrazene-forming reaction has been successfully applied to the site-specific modification of DNA substrates. The high reaction efficiency at a desired location and high tolerance toward a variety of functional groups on azide and phosphine reagents could be of significant help in tailoring the technology to more specific applications. Thanks to the widespread use of azide-alkyne cycloaddition reactions in the chemistry and biology communities, ${ }^{42}$ there are numerous commercially available alkyl azide reagents, and the current research technologies can be readily adopted for diverse applications. The shelf-stable nature of the alkyl azide and triarylphosphine reagents would also be practically helpful in this context. Persistent issues of common aminetargeting reagents originate from instability issues such as the hydrolytic decomposition of $\mathrm{N}$ hydroxysuccinimide (NHS) ester reagents for the acylation reaction and the aerobic oxidation of aldehyde reagents used in the reductive alkylation reaction. Our initial success of ionic-liquid bioconjugation development for nucleotide substrates may serve to provide further access to untapped chemical labeling methodologies for preparation of nucleotide conjugates.

\section{Author Information}

Corresponding Author

Jun Ohata - Department of Chemistry, North Carolina State University, Raleigh, North Carolina 27695, United States; 
orcid.org/0000-0002-3614-7472; Email: johata@ ncsu.edu

\section{Authors}

Seiya Ishizawa - Department of Chemistry, North Carolina State University, Raleigh, North Carolina 27695, United States; orcid.org/0000-0003-1925-031X

Munkhtuya Tumurkhuu - Department of Chemistry, North Carolina State University, Raleigh, North Carolina 27695, United States; orcid.org/0000-0003-4247-4020

Elizabeth J. Gross - Department of Chemistry, North Carolina State University, Raleigh, North Carolina 27695, United States; orcid.org/0000-0003-2153-5381

\section{Conflicts of interest}

The authors declare the following competing financial interest(s): The authors have filed a patent application on the ionic-liquid-based tetrazene-forming bioconjugation method.

\section{Acknowledgement}

This work was financially supported by North Carolina State University. E.J.G. was financially supported by an NC State Provost's Professional Experience Program (PEP). We thank Dr. Alison Killilea and Wilmene Hercule of the UC Berkeley Cell Culture Facility for cell culture support, and Dr. G. W. A. Milne for his editing of our manuscript. Confocal microscopy imaging was performed at the Cellular and Molecular Imaging Facility (CMIF), and MALDI-MS experiments were performed in Molecular Education, Technology and Research Innovation Center (METRIC) at NC State University, which is supported by the State of North Carolina.

\section{References}

1 J. Zheng, R. Yang, M. Shi, C. Wu, X. Fang, Y. Li, J. Li and W. Tan, Chem Soc Rev, 2015, 44, 3036-3055. 2 R. Monroy-Contreras and L. Vaca, J Nucleic Acids, 2011, 2011, 741723.

3 C. J. Smith and A. M. Osborn, FEMS Microbiol Ecol, 2009, 67, 6-20.

4 R. Ting, L. Lermer and D. M. Perrin, J. Am. Chem. Soc., 2004, 126, 12720-12721.

5 X. Wang, M. Feng, L. Xiao, A. Tong and Y. Xiang, ACS Chem. Biol., 2016, 11, 444-451.

6 A. J. Boersma, R. P. Megens, B. L. Feringa and G. Roelfes, Chem. Soc. Rev., 2010, 39, 2083-2092.

7 J. A. Kulkarni, D. Witzigmann, S. B. Thomson, S. Chen, B. R. Leavitt, P. R. Cullis and R. van der Meel, Nat. Nanotechnol., 2021, 16, 630-643.

8 D. D. Young, M. O. Lively and A. Deiters, J. Am. Chem. Soc., 2010, 132, 6183-6193.

9 M. E. Østergaard, C. L. De Hoyos, W. B. Wan, W. Shen, A. Low, A. Berdeja, G. Vasquez, S. Murray, M. T. Migawa, X. Liang, E. E. Swayze, S. T. Crooke and P. P. Seth, Nucleic Acids Research, 2020, 48, 1691-1700.

10 Y. Ma, X. Zhang, X. Xu, L. Shen, Y. Yao, Z. Yang and P. Liu, PLoS One, 2015, 10, e0124924.

11 N. B. Struntz and D. A. Harki, ACS Chem. Biol., 2016, 11, 1631-1638.

12 C. Yu, M. An, M. Li and H. Liu, Mol. Pharmaceutics, 2017, 14, 2815-2823.

13 W. Meng, T. Yamazaki, Y. Nishida and N. Hanagata, BMC Biotechnology, 2011, 11, 88. 
14 R. A. Hughes and A. D. Ellington, Cold Spring Harb Perspect Biol, 2017, 9, a023812.

15 D. Xu, N. Rivas-Bascón, N. M. Padial, K. W. Knouse, B. Zheng, J. C. Vantourout, M. A. Schmidt, M. D. Eastgate and P. S. Baran, J. Am. Chem. Soc., 2020, 142, 5785-5792.

16 K. Tishinov, N. Fei and D. Gillingham, Chem. Sci., 2013, 4, 4401-4406.

17 S. N. Geigle, L. A. Wyss, S. J. Sturla and D. G. Gillingham, Chem. Sci., 2016, 8, 499-506.

18 D. Gillingham and R. Shahid, Curr Opin Chem Biol, 2015, 25, 110-114.

19 E. K. Jang, R. G. Son and S. P. Pack, Nucleic Acids Research, 2019, 47, e102-e102.

20 D. T. Flood, K. W. Knouse, J. C. Vantourout, S. Kitamura, B. B. Sanchez, E. J. Sturgell, J. S. Chen, D. W. Wolan, P. S. Baran and P. E. Dawson, ACS Cent. Sci., 2020, 6, 1789-1799.

21 J. Wang, H. Lundberg, S. Asai, P. Martín-Acosta, J. S. Chen, S. Brown, W. Farrell, R. G. Dushin, C. J. O'Donnell, A. S. Ratnayake, P. Richardson, Z. Liu, T. Qin, D. G. Blackmond and P. S. Baran, PNAS, 2018, 115, E6404-E6410.

22 D. K. Romney, F. H. Arnold, B. H. Lipshutz and C.-J. Li, J. Org. Chem., 2018, 83, 7319-7322.

23 S. K. Shukla and J.-P. Mikkola, Front. Chem., 2020, 8, 1219.

24 D. R. MacFarlane, A. L. Chong, M. Forsyth, M. Kar, R. Vijayaraghavan, A. Somers and J. M. Pringle, Faraday Discuss., 2017, 206, 9-28.

25 H. M. El-Shaffey, E. J. Gross, Y. D. Hall and J. Ohata, J. Am. Chem. Soc., 2021, 143, 12974.

26 Q. Liu, Q. He, X. Lyu, F. Yang, Z. Zhu, P. Xiao, Z. Yang, F. Zhang, Z. Yang, X. Wang, P. Sun, Q. Wang, C. Qu, Z. Gong, J. Lin, Z. Xu, S. Song, S. Huang, S. Guo, M. Han, K. Zhu, X. Chen, A. W. Kahsai, K.-H. Xiao, W. Kong, F. Li, K. Ruan, Z. Li, X. Yu, X. Niu, C. Jin, J. Wang and J. Sun, Nat Commun, 2020, 11, 4857.

27 W. Hu, H. Wang, Y. Hou, Y. Hao and D. Liu, FEBS Letters, 2019, 593, 1113-1121.

28 P. Gao, W. Pan, N. Li and B. Tang, Chem. Sci., 2019, 10, 6035-6071.

29 X. Wu, X. He, L. Zhong, S. Lin, D. Wang, X. Zhu and D. Yan, J. Mater. Chem., 2011, 21, 1361113620.

30 S. Sun, Y. Cao, J. Feng and P. Wu, J. Mater. Chem., 2010, 20, 5605-5607.

31 A. Nantalaksakul, A. Mueller, A. Klaikherd, C. J. Bardeen and S. Thayumanavan, J. Am. Chem. Soc., 2009, 131, 2727-2738.

32 N. Kuntip, D. Japrung and P. Pongprayoon, Biopolymers, 2021, 112, e23421.

33 B. Stewart, A. Harriman and L. J. Higham, Organometallics, 2011, 30, 5338-5343.

34 J. Kiernan, Biotechnic \& Histochemistry, 2018, 93, 133-148.

35 Z. Cao, R. Tong, A. Mishra, W. Xu, G. C. L. Wong, J. Cheng and Y. Lu, Angewandte Chemie International Edition, 2009, 48, 6494-6498.

36 Y. Wan, L. Wang, C. Zhu, Q. Zheng, G. Wang, J. Tong, Y. Fang, Y. Xia, G. Cheng, X. He and S.Y. Zheng, Cancer Res, 2018, 78, 798-808.

37 D. Sekkai, E. Dausse, C. Di Primo, F. Darfeuille, C. Boiziau and J.-J. Toulmé, Antisense and Nucleic Acid Drug Development, 2002, 12, 265-274.

38 A. Ohmann, K. Göpfrich, H. Joshi, R. F. Thompson, D. Sobota, N. A. Ranson, A. Aksimentiev and U. F. Keyser, Nucleic Acids Research, 2019, 47, 11441-11451.

39 S. F. Jones, H. Joshi, S. J. Terry, J. R. Burns, A. Aksimentiev, U. S. Eggert and S. Howorka, J. Am. Chem. Soc., 2021, 143, 8305-8313.

$40 \quad$ N. Iqbal and N. Iqbal, Molecular Biology International, 2014, 2014, e852748.

41 G. Mahlknecht, R. Maron, M. Mancini, B. Schechter, M. Sela and Y. Yarden, PNAS, 2013, 110, 8170-8175.

42 N. Z. Fantoni, A. H. El-Sagheer and T. Brown, Chem. Rev., 2021, 121, 7122-7154. 